\title{
Unsteady Force Measurements in Pitching-Plunging Airfoils
}

\author{
Luis P Bernal ${ }^{1}$ \\ University of Michigan, Ann Arbor, MI 48109, U.S.A \\ Michael V. $\mathrm{Ol}^{2}$, Douglas P. Szczublewski ${ }^{3}$ and Craig A. Cox ${ }^{4}$ \\ U.S. Air Force Research Laboratory, Wright-Patterson Air Force Base, OH 45433, U.S.A.
}

\begin{abstract}
A new sensor for unsteady force measurements of pitching and plunging airfoils using Fiber Bragg Gratings (FBG) in a water channel facility has been the developed. Load cells having four or five FBG sensors written in a single optical fiber have been developed to measure the strain at flexures designed and determine the normal and axial forces, the pitching moment and the temperature. The main advantage of the present approach compared to other designs using electrical strain gages is the simplicity of a single optical fiber carrying all the sensor information, and low noise. The design, compensation, calibration and application to force measurement on an airfoil at steady and unsteady flow conditions are described. The measurement results show the unsteady large lift coefficients produced during mild and deep stall in a pitching and plunging SD 7003 airfoil.
\end{abstract}

\section{Nomenclature}

A

$C_{L}$

$c$

$f$

$h$

$h_{0}$

$k$

$\mathrm{M}$

$\mathrm{N}$

$\mathrm{n}_{\text {eff }}$

$\mathrm{P}_{\mathrm{e}}$

Re

St

$t / T$

$T$

$\mathrm{T}$

$U_{\infty}$

$\alpha$

$\alpha_{0}$

$\alpha_{e}$

$\alpha_{\mathrm{s}}$

$\alpha_{\mathrm{f}}$

$\Delta \mathrm{T}$

$\Delta \lambda_{\mathrm{A}}$
$=$ axial force, in $\mathrm{lbf}$

$=$ airfoil section lift coefficient

$=$ airfoil chord $(=152.4 \mathrm{~mm})$

$=$ airfoil oscillation pitching/plunging frequency

$=$ plunging position as function of time measured at the center of rotation of the airfoil

$=$ non-dimensional plunging amplitude

$=$ reduced frequency of pitch or plunge, $k=2 \pi f c /\left(2 U_{\infty}\right)$

$=$ pitching moment relative to the center of the load cell, in in-lbf

$=$ normal force, in $\mathrm{lbf}$

$=$ effective optical fiber refractive index

$=$ strain-optic coefficient

$=$ Reynolds number, $R e=U_{\infty} c / v, v$ taken as $10^{-6}$ in SI units for water at $20^{\circ} \mathrm{C}$

$=$ Strouhal number, $S t=2 f c h_{0} / U_{\infty}=2 k h_{0} / \pi$

$=$ dimensionless time, in fractions of one oscillation period

$=$ airfoil oscillation period, $T=1 / f$

$=$ temperature, in ${ }^{\circ} \mathrm{F}$

$=$ free stream (reference) velocity

$=$ kinematic angle of incidence due to pitch (pitch angle)

$=$ mean angle of attack (that is, the constant pitch angle offset from zero)

$=$ total angle of attack from trigonometric combination of pitch and plunge

$=$ thermal expansion coefficients of the fiber bonding material

$=$ thermal expansion coefficients of the fiber

$=$ temperature change

= wavelength shift produced by axial force $\mathrm{A}$, in $\mathrm{nm}$

${ }^{1}$ Associate Professor, University of Michigan, Department of Aerospace Engineering, lpb@umich.edu.

${ }^{2}$ Aerospace Engineer, Air Vehicles Directorate, Wright-Patterson AFB, Michael.Ol@wpafb.af.mil.

${ }^{3}$ Air Vehicles Directorate, Wright-Patterson AFB, Douglas .Szczublewski@wpafb.af.mil.

${ }^{4}$ Air Vehicles Directorate, Wright-Patterson AFB, Craig.Cox@wpafb.af.mil.

American Institute of Aeronautics and Astronautics 


\begin{tabular}{|c|c|}
\hline$\Delta \lambda_{\mathrm{B}}$ & $=\mathrm{FBG}$ wavelength shift \\
\hline$\Delta \lambda_{\mathrm{i}}$ & $=$ wavelength shift of FBG number i $(\mathrm{i}=1-4)$, in $\mathrm{nm}$ \\
\hline$\Delta \lambda_{\mathrm{M}}$ & $=$ wavelength shift produced by pitching moment $\mathrm{M}$, in $\mathrm{nm}$ \\
\hline$\Delta \lambda_{\mathrm{N}}$ & $=$ wavelength shift produced by normal force $\mathrm{N}$, in $\mathrm{nm}$ \\
\hline$\Delta \lambda_{\mathrm{T}}$ & $=$ wavelength shift produced by temperature $\mathrm{T}$, in $\mathrm{nm}$ \\
\hline$\Delta \varepsilon$ & $=$ strain change acting on the fiber \\
\hline$\phi$ & $=$ phase difference between pitching and plunging; positive $\rightarrow$ pitch leads \\
\hline$\Lambda$ & $=$ period of the FBG refractive index modulation \\
\hline$\lambda$ & $=$ ratio of pitch-amplitude to plunge-induced angle of attack \\
\hline$\lambda_{\mathrm{B}}$ & $=\mathrm{FBG}$ wavelength \\
\hline$\theta_{o}$ & $=$ amplitude of the airfoil pitch oscillation \\
\hline$\zeta$ & $=$ fiber thermo-optic coefficient \\
\hline
\end{tabular}

\section{Introduction}

$\mathrm{T}$ HIS paper reports the development of an unsteady force measurement system to determine the force on an airfoil in Pitching-Plunging motion. The research is motivated by recent interest in the aerodynamics of flapping wings found in nature. These are biological examples of integrated lift and propulsive systems that could offer new solutions for very small Micro Air Vehicle (MAV) systems. Central to the performance of these systems is force generation, and the relation of the force produced to the kinematics of the motion and associated flow features. Research in the last few years using Particle Image Velocimetry (PIV), flow visualization as well as computational techniques has documented the development of separated flow regions and vortical structures which are believed to contribute to the development of aerodynamic force on the airfoil ${ }^{1,2}$. However the connection between unsteady aerodynamic force and flow structure remains tenuous because of a lack of direct time-resolved force measurements in these flows. In the present research we extend Particle Image Velocimetry flow measurements and flow visualization results to include direct force measurements, and thus provide a more comprehensive view of the unsteady aerodynamics of pitching and plunging airfoils.

Measurements of the mean force acting on a pitching and plunging NACA 0012 airfoil were reported by Anderson et al. ${ }^{3}$ who showed that maximum propulsion efficiency is obtained at a Strouhal number between 0.1 and 0.3 and for combined pitch/plunge motion. More recently Heathcote $e t a l .{ }^{4}$ report mean and instantaneous trust measurements on rigid and flexible airfoils in pure plunge motion. These results also show an optimum Strouhal number for propulsion efficiency. Their results also show that a moderate amount of flexibility also improves propulsion efficiency. However these investigations do not report lift and drag measurements as a function of pitch angle and therefore the role of vortical features on force development cannot be determined directly. Force measurements obtained with the present instrumentation have been reported by $\mathrm{Ol}$ et.al. ${ }^{5}$. In the present paper we provide a detailed description of the design, calibration and measurement procedures used for the force measurements and provide additional insight on the prospect for this technology.

\section{Flow Facility and Force Measurement Instrumentation}

\section{A. Flow Facility}

The force sensor based on Fiber Bragg Grating (FBG) technology has been developed for use in the Air Force Research Laboratory Air Vehicles Directorate's Horizontal Free-surface Water Tunnel (HFWT), Wright Patterson Air Force Base. The load cell is an integral part of the facility, and the design was optimized to minimize changes to the facility or model positioning hardware. A picture of the flow facility is shown in Figure 1a. The test section is $0.46 \mathrm{~m} \times 0.61 \mathrm{~m} \times 2.74 \mathrm{~m}$. The airfoil motion is produced by a 2-degree-of-freedom pitch-plunge rig, consisting of a pair of electric linear servomotors mounted vertically on a plate above the tunnel test section free-surface. The model is supported along its midspan by two vertical rods and mounted upside down as shown in Figure 1b. Each motor actuates a vertical rod, which connects via a bushing to a mounting plate which in turn is attached to the airfoil. Motion trajectory of each rod is programmed independently, allowing for single degree-of-freedom motions such as sinusoidal pure-pitch or pure-plunge, as well as non-trigonometric and combined motions. Figure 1c shows the airfoil model installation in the water tunnel with the vertical rods attached to the mounting plate as indicated. 
Also shown in Figure 1c is the location of the mounting plate used to attach the airfoil to the plunge rods. For the force measurement the mounting plate is replaced by a load cell which incorporated the same functionality as the attachment plate in terms of airfoil motion kinematics and includes the FBG strain sensors required for the measurements as described below.

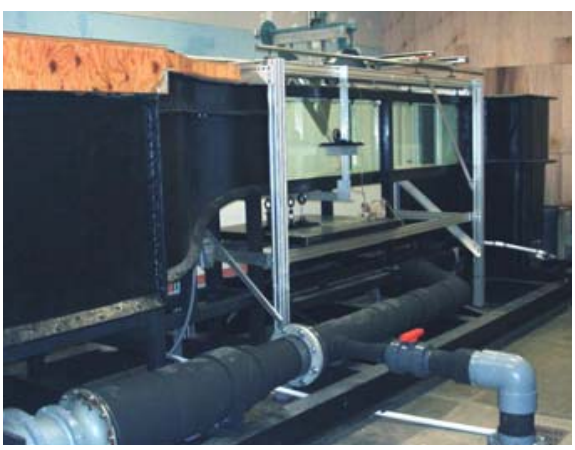

(a)

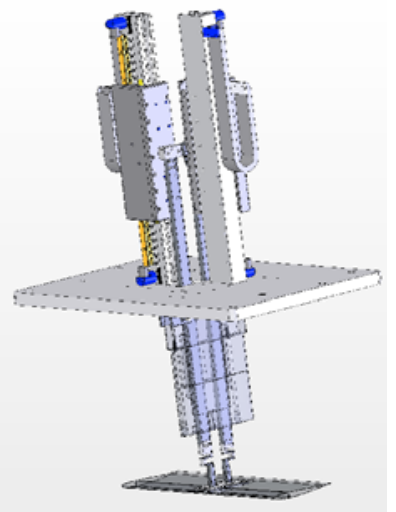

(b)

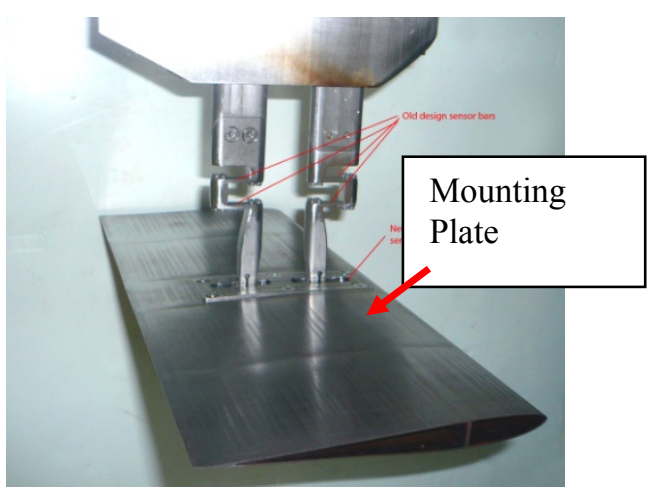

(c)

Figure 1. AFRL Horizontal Free-surface Water Tunnel (left); schematic of pitch/plunge rig and airfoil model (middle); and SD7003 airfoil installed in HFWT test section, with plunge rods in position (right).

\section{B. Basic Principles of Fiber Bragg Gratings Technology}

In here we provide a brief summary of FBG technology as relevant to present application. A more detailed description of the technology can be found in the review paper by Zhang et al. ${ }^{6}$. A FBG sensor is a single-mode optical fiber in which a periodic index-of-refraction modulation along the fiber direction (grating) is introduced in a short section of the fiber. Light propagating along the fiber refracts in the grating resulting in a reflected signal of very narrow wavelength determined by the period of the refractive index modulation. The wavelength of the reflected signal is given by

$$
\lambda_{\mathrm{B}}=2 \mathrm{n}_{\text {eff }} \Lambda
$$

where $\mathrm{n}_{\text {eff }}$ is the effective refractive index of the single-mode optical fiber, and $\Lambda$ is the period of the refractive index modulation. In typical implementations of the technology, the gratings are 8 to $20 \mathrm{~mm}$ long and several can be placed along the same fiber spaced from centimeters to a few meters apart.

FBG sensors measure strain by detecting very small changes in the wavelength of the reflected light. The change in wavelength, $\Delta \lambda_{B}$ is a function of the strain and the temperature change in the fiber,

$$
\frac{\Delta \lambda_{\mathrm{B}}}{\lambda_{\mathrm{B}}}=\mathrm{P}_{\mathrm{e}} \Delta \varepsilon+\left[\mathrm{P}_{\mathrm{e}}\left(\alpha_{\mathrm{s}}-\alpha_{\mathrm{f}}\right)+\varsigma\right] \Delta \mathrm{T}
$$

where $\mathrm{P}_{\mathrm{e}}$ is the strain-optic coefficient, $\Delta \varepsilon$ is the strain acting on the fiber, $\alpha_{\mathrm{s}}$ and $\alpha_{\mathrm{f}}$ are the thermal expansion coefficients of the fiber bonding material and the fiber, respectively, and $\zeta$ is the thermo-optic coefficient. The normalized constant temperature strain response is,

$$
\frac{\Delta \lambda_{\mathrm{B}}}{\lambda_{\mathrm{B}} \Delta \varepsilon}=0.78 \times 10^{-6} 1 / \text { micro }- \text { strain }
$$

and the normalized thermal response at constant strain is,

$$
\frac{\Delta \lambda_{\mathrm{B}}}{\lambda_{\mathrm{B}} \Delta \mathrm{T}}=6.678 \times 10^{-6}\left({ }^{\circ} \mathrm{C}\right)^{-1} \text {. }
$$

For typical commercial FBS sensors the wavelength shifts are $\sim 1 \mathrm{pm} /$ micro-strain and $10 \mathrm{pm} /{ }^{\circ} \mathrm{C}\left(1 \mathrm{pm}=10^{-12} \mathrm{~m}\right)$.

The second important element of an FBG system is the wavelength reading instrument or optical sensor interrogator. A variety of techniques have been developed (see Zhang et al. ${ }^{6}$ ), with some of them available commercially. A typical commercial system has the capability to sample multiple channels (4), each having multiple SBG sensors (100s) with resolution as small as $0.1 \mathrm{pm}$ and sampling frequency up to a few kHz. These performance 
metrics make the technology very attractive for the present application. Key features that are particularly relevant are: 1) the sensing element is optical and should be immune to em-radiation and other noise sources; 2) very small sensors (the size of the optical fiber) that could be easily integrates into the load cell structure. Important challenges for the technology are: 1) high temperature sensitivity that will require temperature compensation, and 2) fiber optic bending radius should be larger 1-inch which may represent a problem for very small systems. These challenges must be adequately addressed in the design of the load cell to achieve the high sensitivity and balance stiffness required for the present application.

\section{Load Cell Mechanical Design}

FBGs measure strain at a specific location in an optical fiber by detecting the spectrum of reflected light. The fiber reflection spectrum has a maximum at a wavelength which is proportional to the local strain at the location of a FBG sensor. As noted above the main advantages of the technology relevant to the present application are: 1 - It is an optical sensing technique and therefore immune to electronic noise, which is particularly problematic for electronic sensors in water and in proximity to the high current linear motors used to drive the model; 2 - Several FBG strain sensors can be placed in the same fiber at precise locations and, therefore, only a single fiber is needed for a multi component force sensor. For the present research the mounting plate was instrumented with $4\left(1^{\text {st }}\right.$ generation) or 5 ( $2^{\text {nd }}$ generation) FBG sensors to measure the lift, drag and pitching moment acting on the airfoil. The basic geometry of the mounting plate is the same as the original mounting plate. The plunge rods pivot locations, airfoil model attachment points and thickness are the same. The flexures and fiber optic paths are machined to accommodate the FBG sensors. A drawing of the $1^{\text {st }}$ generation instrumented mounting plate is shown in Figure 2. There are two parts in the modified attachment plate. The plunge rods are attached to the center part of the plate at the pivot points, while the airfoil model is attached to the outer part of the plate. Thin flexures instrumented with FBG strain sensors join the two parts of the plate. The locations of the FBGs are designed to provide temperature compensation as well as decoupling between the lift, drag and pitching moment component measurements as discussed below.

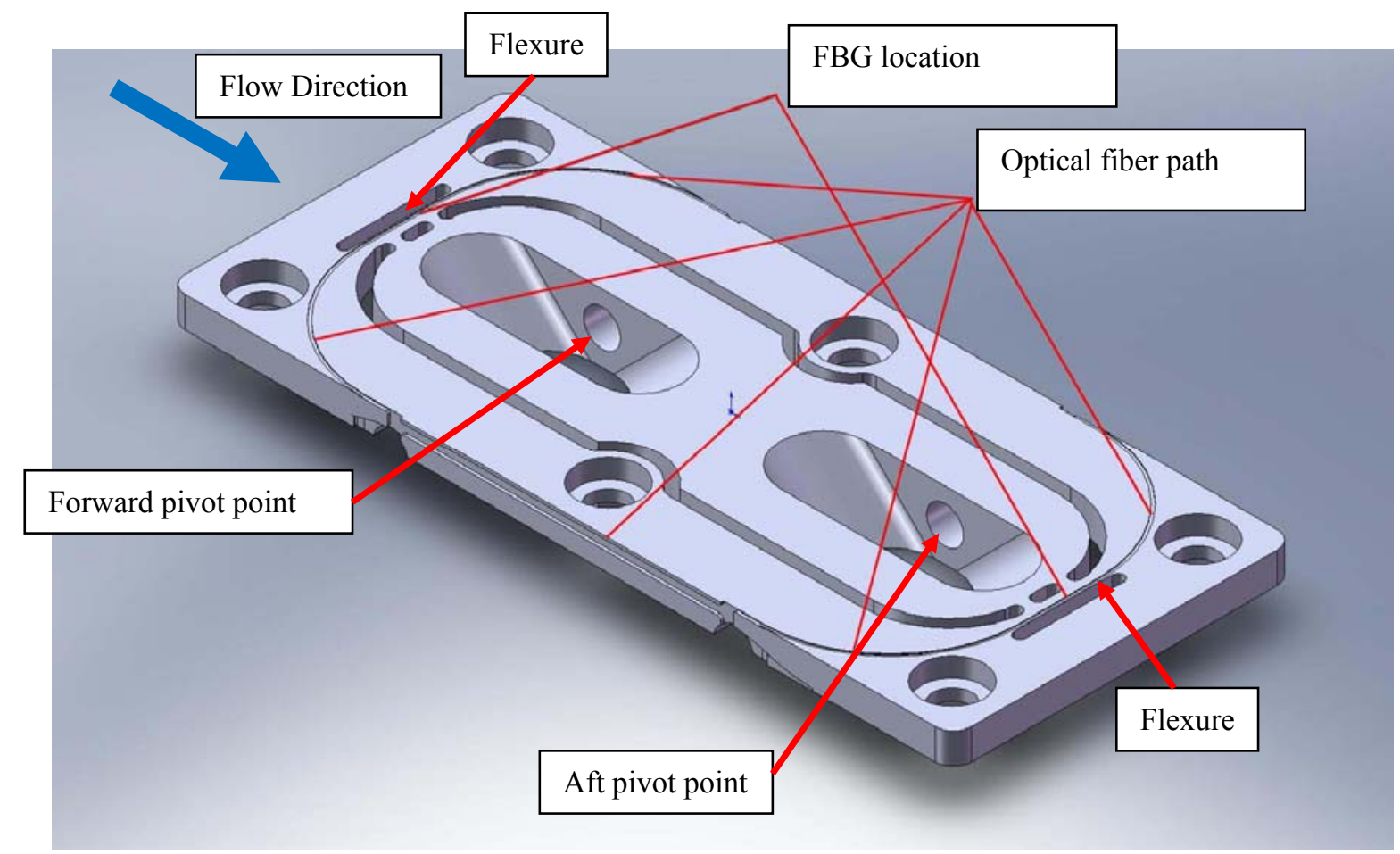

Figure 2. Drawing of the mounting plate showing the optical fiber path and the location of the flexures and FBG sensors on the top surface of the plate. Another two FBG sensors are placed on the bottom side of the flexures.

4

American Institute of Aeronautics and Astronautics 
A commercial CAD package with FEM analysis (SolidWorks, Dassault Systèmes SolidWorks Corp., http://www.solidworks.com/) was used to size the flexures and to obtain preliminary estimates of the load cell calibration matrix and stiffness characteristics. The primary design parameters are the flexures length and cross section dimensions; and the spacing between the two attachment points of the load cell center section to the flexures. For the data reported here the flexure lengths are 0.75 ", the cross sections are $0.033 " \times 0.140 "$ and the distances between center section attachment points are $0.375 "$. The resonance frequency of the load cell model combination with the SD7003 model mounted was estimated using FEM analysis and is approximately $60 \mathrm{~Hz}$.

\section{Load Cell Compensation and Calibration}

The load cell is designed to measure the force components normal to the mounting plate, parallel to the plate (axial force) and the pitching moment. The four FBG sensors in the fiber are fabricated to reflect light of a specific wavelength and located in the load cell at specific locations given in Table 1. During installation the FBG sensors are pre-strained before bonding to the flexures. The resulting shift in wavelength for no load is also given in Table 1.

Table 1. Location and Wavelength of FBG Sensors in Load Cell

\begin{tabular}{|c|c|c|c|}
\hline FBG \# & Location & Wavelength (nm) & No Load Wavelength (nm) \\
\hline 1 & Bottom Aft & 1526 & 1526.833 \\
\hline 2 & Bottom Forward & 1536 & 1536.765 \\
\hline 3 & Top Aft & 1546 & 1546.788 \\
\hline 4 & Top Forward & 1556 & 1556.607 \\
\hline
\end{tabular}

An important feature of the design is that the load cell sensors outputs can be combined to decouple the tree force components and a temperature output. A positive normal force (towards the suction side of the airfoil) produces positive strain in the FBGs mounted on the top of the sensor bars and negative strain on the FBGs mounted on the bottom of the sensor bars. Similarly positive axial force (dowstream) produces positive strain in the FBG mounted on the forward sensor bar and negative strain in the FBG mounted on the aft sensor bar. A positive pitching moment (forward up) produces positive strain in top forward and bottom aft FBGs and negative strain in the bottom forward and top aft FBGs. And an increase in temperature produces a positive increase of the wavelength of all the FBG sensors. Hence, for the purpose of calibration we define,

$$
\begin{gathered}
\Delta \lambda_{\mathrm{N}}=-\Delta \lambda_{1}-\Delta \lambda_{2}+\Delta \lambda_{3}+\Delta \lambda_{4} \\
\Delta \lambda_{\mathrm{A}}=\Delta \lambda_{1}-\Delta \lambda_{2}+\Delta \lambda_{3}-\Delta \lambda_{4} \\
\Delta \lambda_{\mathrm{M}}=\Delta \lambda_{1}-\Delta \lambda_{2}-\Delta \lambda_{3}+\Delta \lambda_{4} \\
\Delta \lambda_{\mathrm{T}}=\Delta \lambda_{1}+\Delta \lambda_{2}+\Delta \lambda_{3}+\Delta \lambda_{4}
\end{gathered}
$$

or in matrix form,

$$
\left(\begin{array}{l}
\Delta \lambda_{\mathrm{N}} \\
\Delta \lambda_{\mathrm{A}} \\
\Delta \lambda_{\mathrm{M}} \\
\Delta \lambda_{\mathrm{T}}
\end{array}\right)=\left(\begin{array}{cccc}
-1 & -1 & 1 & 1 \\
-1 & 1 & -1 & 1 \\
1 & -1 & -1 & 1 \\
1 & 1 & 1 & 1
\end{array}\right)\left(\begin{array}{l}
\Delta \lambda_{1} \\
\Delta \lambda_{2} \\
\Delta \lambda_{3} \\
\Delta \lambda_{4}
\end{array}\right)
$$

Then, in the calibration matrix is

$$
\left(\begin{array}{c}
\mathrm{N} \\
\mathrm{A} \\
\mathrm{M} \\
\mathrm{T}
\end{array}\right)=\left(\begin{array}{llll}
\mathrm{C}_{11} & \mathrm{C}_{12} & \mathrm{C}_{13} & \mathrm{C}_{14} \\
\mathrm{C}_{21} & \mathrm{C}_{22} & \mathrm{C}_{23} & \mathrm{C}_{24} \\
\mathrm{C}_{31} & \mathrm{C}_{32} & \mathrm{C}_{33} & \mathrm{C}_{34} \\
\mathrm{C}_{41} & \mathrm{C}_{42} & \mathrm{C}_{43} & \mathrm{C}_{44}
\end{array}\right)\left(\begin{array}{c}
\Delta \lambda_{\mathrm{N}} \\
\Delta \lambda_{\mathrm{A}} \\
\Delta \lambda_{\mathrm{M}} \\
\Delta \lambda_{\mathrm{T}}
\end{array}\right)+\left(\begin{array}{c}
\mathrm{N}_{\mathrm{o}} \\
\mathrm{A}_{\mathrm{o}} \\
\mathrm{M}_{\mathrm{o}} \\
\mathrm{T}_{\mathrm{o}}
\end{array}\right) .
$$


Where the calibration matrix is expect to be almost diagonal.

In a typical calibration, the load cell is mounted on horizontal plane and at angles of $\pm 45^{\circ}$; and loaded with weights in the range 0 to $5 \mathrm{lbf}$. At each load condition the wavelength shifts $\Delta \lambda_{\mathrm{i}}, \quad \mathrm{i}=1-4$ are measured and the values of $\Delta \lambda_{\mathrm{N}}, \Delta \lambda_{\mathrm{A}}, \Delta \lambda_{\mathrm{M}}$, and $\Delta \lambda_{\mathrm{T}}$ are calculated. A least squares fit to the data gives the calibration matrix,

$$
\left(\begin{array}{c}
\mathrm{N} \\
\mathrm{A} \\
\mathrm{M} \\
\mathrm{T}
\end{array}\right)=\left(\begin{array}{cccc}
58.477 & 7.457 & 4.457 & 0 \\
1.251 & 137.009 & 1.190 & 0 \\
0.141 & 2.965 & 104.960 & 0 \\
-2.413 & -3.4 & -9.235 & 8.813
\end{array}\right)\left(\begin{array}{l}
\Delta \lambda_{\mathrm{N}} \\
\Delta \lambda_{\mathrm{A}} \\
\Delta \lambda_{\mathrm{M}} \\
\Delta \lambda_{\mathrm{T}}
\end{array}\right)+\left(\begin{array}{c}
0.030 \\
0.011 \\
-0.022 \\
50.854
\end{array}\right) .
$$

where the wavelength shift are expressed in $\mathrm{nm}$, the physical variables $(\mathrm{N}$ - normal force, $\mathrm{A}$ - axial force, $\mathrm{M}$ pitching moment and $\mathrm{T}$ - temperature) are expressed in imperial units.

Standard error for the force and temperature calibration results are given in Table 2 and plots of the calibration data are presented in Figure 3. The calibration matrix diagonal elements for the force components are very large compared to the off diagonal elements indicating good decoupling between the measured force components and good temperature compensation. This is also shown by the plots which show excellent correlation between the force applied and component wavelength shift.

\section{Table 2. Load Cell Calibration Standard Error}

\begin{tabular}{|c|c|}
\hline & Standard Error \\
\hline $\mathrm{N}(\mathrm{lbf})$ & 0.11 \\
\hline $\mathrm{A}(\mathrm{lbf})$ & 0.15 \\
\hline $\mathrm{M}$ (in-lbf) & 0.07 \\
\hline $\mathrm{T}(\mathrm{K})$ & 0.34 \\
\hline
\end{tabular}
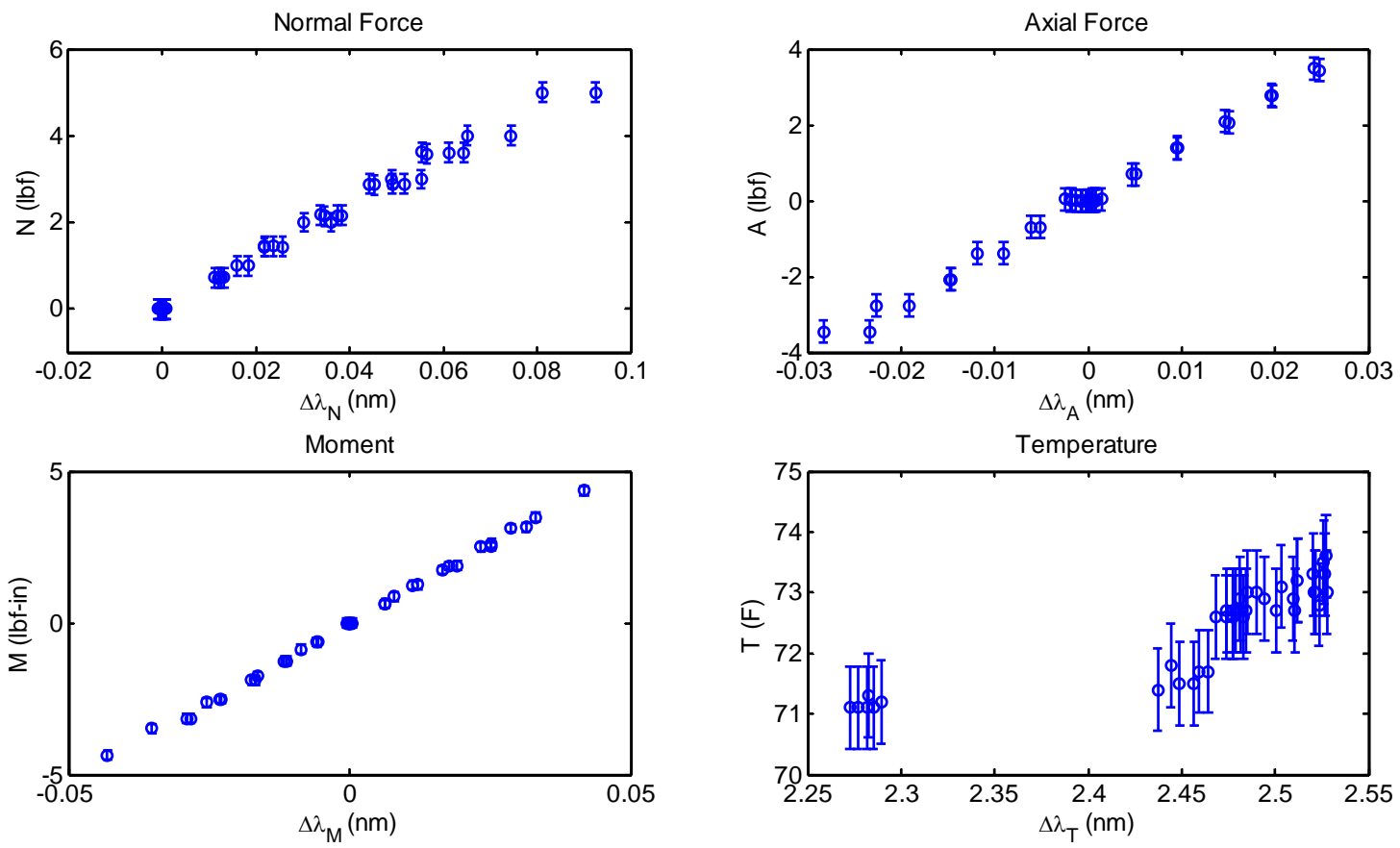

Figure 3. Load cell calibration results. The error bars show the $95 \%$ confidence interval for each measurement.

6

American Institute of Aeronautics and Astronautics 


\section{E. FBG Data Processing}

A Micron Optics si425 Optical Sensor Interrogator was used to record the FBG's wavelength information. The instrument was configured to record the output of all 4 FBG sensors in the fiber at a sampling rate of $250 \mathrm{~Hz}$. This sampling rate is much higher than necessary for the present experiments where typical motion frequency is of the order of $0.2 \mathrm{~Hz}$. The data were smoothed using a recursive low pass filter with a cut-off frequency of $6.5 \mathrm{~Hz}(-3 \mathrm{~dB}$ point), which is well above the airfoil motion frequency and the characteristic flow frequency $\mathrm{U} / \mathrm{c} \sim 2.7 \mathrm{~s}^{-1}$. This filter effectively averages data 50 consecutive data points with weight factors designed to optimize high-frequency roll off and it is implemented with symmetric impulse response to eliminate phase distortion. The frequency cut-off of the filter was determined based on measurements of the power spectra of the FBG outputs. Typical power spectra before and after filtering are plotted in Figure 4. Figure 4(a) shows the power spectra of the output data from a typical unsteady run. At low frequency (i.e. $<5 \mathrm{~Hz}$ ) there well-defined spectral peaks associated with the airfoil motion. There is also a very strong peak at $25 \mathrm{~Hz}$. It was determine that this spectral peak is associated with the water channel pump rotational speed. Examination of the flow with flow visualization revealed no evidence of flow structure at this frequency. The power spectrum after filtering the data is shown in Figure 4(b), the peak at $25 \mathrm{~Hz}$ is still present but the magnitude is significantly reduced, to a value comparable to the noise floor of the present measurements $(\sim-5 \mathrm{~dB} / \mathrm{Hz})$.

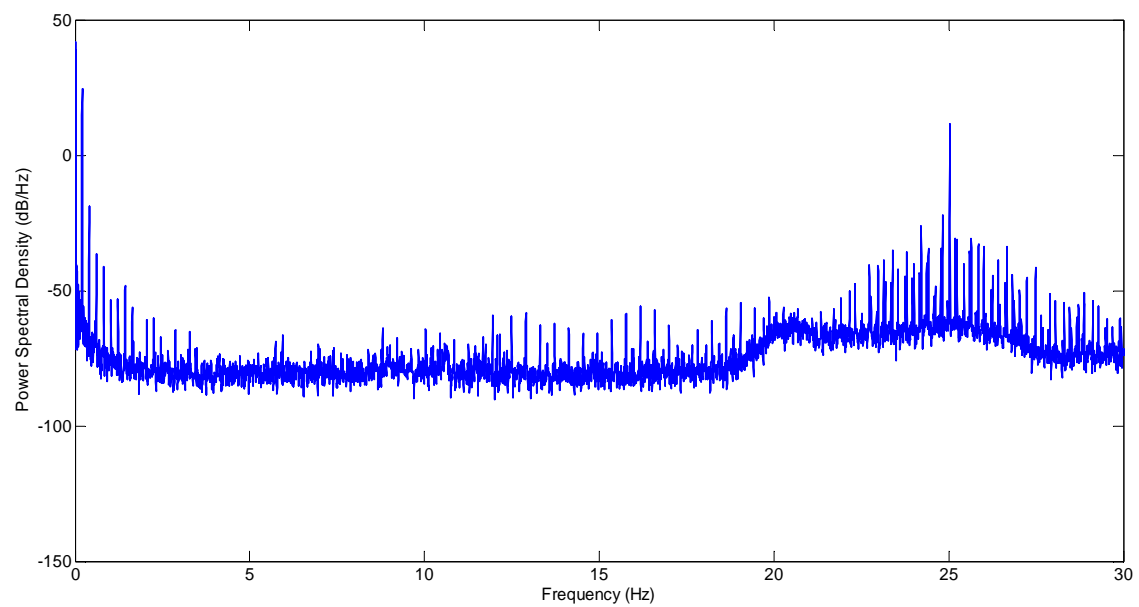

(a)

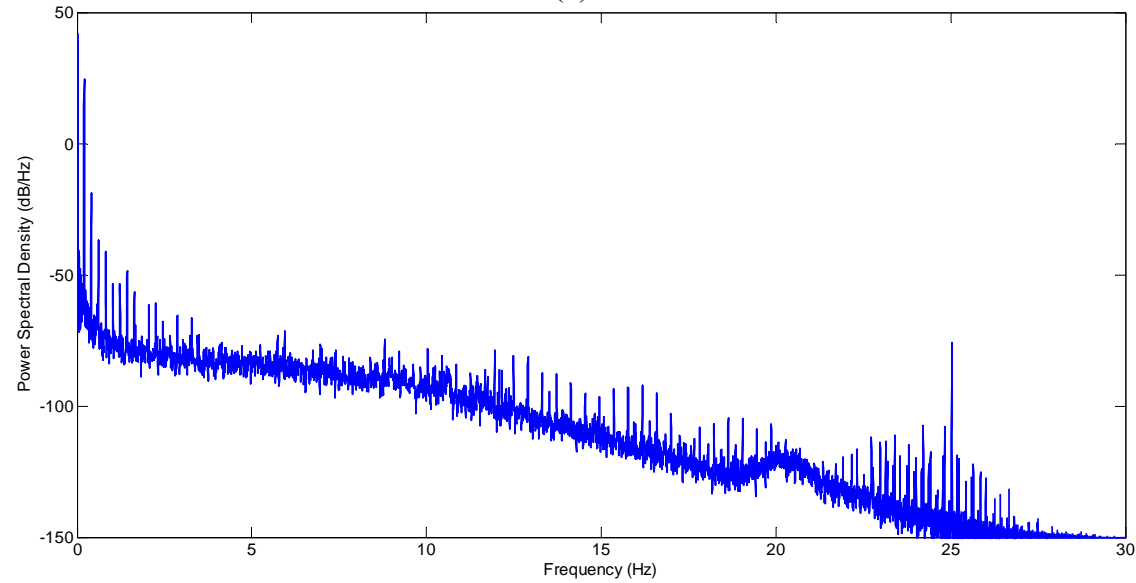

(b)

Figure 4. Typical powers spectra of a FBG sensor output. (a) Spectra of the raw data for a typical run, (b) Spectra after low pass filter. 


\section{Results and Discussion}

\section{A. Force Measurements in Steady Flow}

In order to gain some confidence on the performance of the load cell, steady force measurements were conducted on a SD7003 airfoil at a Reynolds number of 60,000. The steady flow about a SD7003 airfoil at low Reynolds number has been the subject of several recent investigations including in the present flow facility. ${ }^{7}$ The steady flow measurements were conducted in the same apparatus as the unsteady force measurements reported below. The pitch-plunge rig was programmed to position the airfoil at several angles of attack in the range $-12^{\circ}$ to $22^{\circ}$. Data were recorded at each angle of for 10 seconds at a sampling rate of $250 \mathrm{~Hz}$.

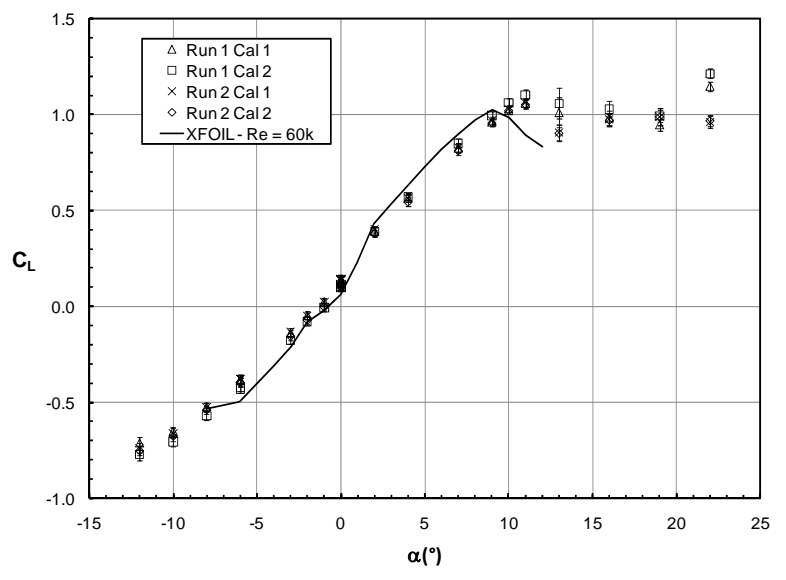

(a)

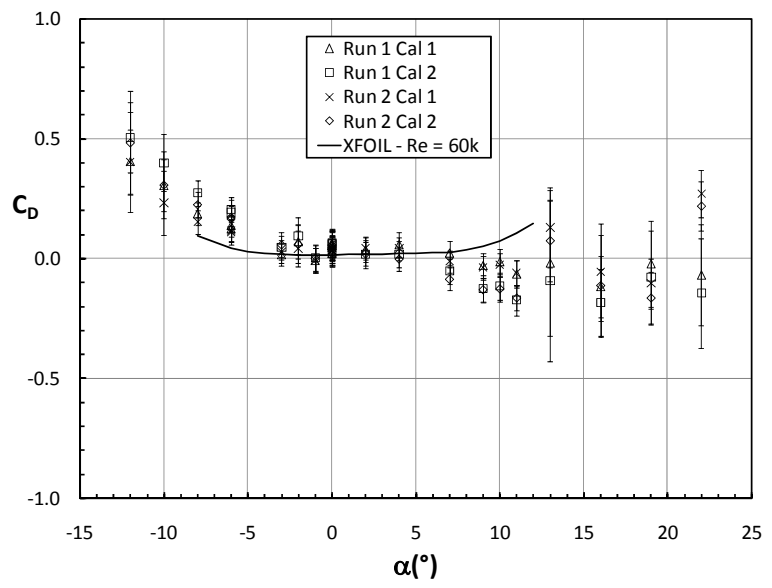

(b)

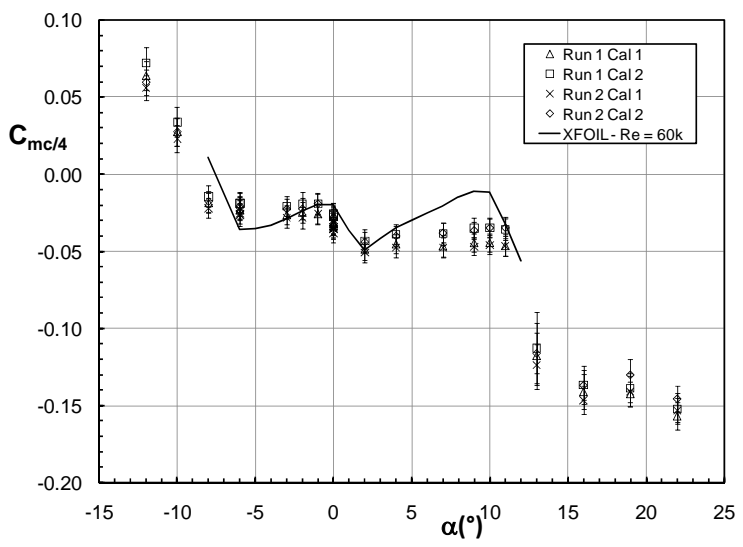

(c)

Figure 5. Force measurements results for SD7003 airfoil at $\mathrm{Re}=\mathbf{6 0 , 0 0 0}$. (a) Lift coefficient, (b) Drag coefficient and (c) Pitching moment coefficient. The error bars are estimates of the $95 \%$ confidence interval for each measurement.

Figure 5 shows the results of these measurements. The error bars are estimates of the $95 \%$ confidence. The experiment was repeated in two independent runs shown labeled as Run1 and 2 in the plots, and the data were processed using two calibration matrices measured on different days labeled as Cal 1 and 2 in the plots. Figure 5(a) plots the section lift coefficient as a function of angle of attack. Also shown in the figure are XFOIL prediction for thSD7003 at $\mathrm{Re}=60,000$ with transition parameter $\mathrm{N}=9$ There is excellent agreement between the two runs and the two calibration matrices. Comparison shows small deviations. The measured results suggest a slightly larger stall angle of attack for both positive and negative angles of attack. Also the maximum lift coefficient measured is larger than the XFOIL result. These discrepancies could be attributed to blockage effects (See also Ol et $a l^{5}$ ). The results 
for the drag coefficient are shown in Figure 5 (b). In this case measurement error is significantly larger than for the lift force giving unreliable results particularly at large angles of attack. The large measurement error for drag is directly related to the large balance stiffness in axial direction as indicated by the large coefficient $C_{22}=137$ of the calibration matrix. Figure 5(c) plots the results for the pitching moment coefficient at the quarter chord point. In this case the agreement is again excellent and the $95 \%$ confidence interval relatively small. The pitching moment data show clearly the onset of stall by the rapid decrease (increase) of the pitching moment at positive (negative) angles of attack. The pitching moment values are in reasonable agreement with XFOIL results, although the increase in pitching moment prior to stall at positive angles of attack is not found in the experimental results.

\section{B. Force Measurements in Pitching Plunging Airfoils}

The main objective of the present research is measurement of the unsteady force on pitching and plunging airfoils. In particular we focus on force measurement on a SD7003 airfoil, which has also been study extensively in unsteady flow conditions at low Reynolds number $(\sim 60,000){ }^{2,5}$ The motion kinematics is described by the pitch and the plunge motion time histories given by

$$
\alpha(t)=\alpha_{o}+\theta_{o} \cos (2 \pi(f t+\varphi)) \quad h(t)=h_{o} \cos (2 \pi f t)
$$

where $\alpha$ is the angle of attack measured relative to the incoming free stream with velocity, $\mathrm{U}_{\infty}, \alpha_{o}$ is the mean angle of attack, $\theta_{o}$ is the amplitude of the pitch oscillation, $h$ is the location of the center of rotation of the airfoil measured normal to the free stream, $h_{o}$ is the normalized amplitude of the plunge motion, $\varphi$ is the phase of the pitch relative to the plunge motions, $f$ is the motion frequency, and $c$ is the airfoil chord. The effective angle of attack, $\alpha_{e}$, is the angle of attack in a frame of reference moving with the center of rotation of the airfoil. For the present kinematics the effective angle of attack is given by

$$
\alpha_{e}(t)=\alpha_{o}+\lambda \arctan (\pi S t) \cos (2 \pi(f t+\varphi))+\arctan (\pi S t \sin (2 \pi f t))
$$

where $S t=2 f c h_{0} / U_{\infty}=2 k h_{0} / \pi$ is the Strouhal number; and $\lambda$ is the ratio of the maximum effective angles of attack of the pitch motion, given by the second term, to the plunge motion, given by the third term. Two airfoil motions kinematics are considered here: a combined pitch-plunge airfoil motion, and a pure plunge motion. The parameters for each case are listed in Table 3.

Table 3. Pitch-Plunge Motion Parameters

\begin{tabular}{|c|c|c|}
\hline & Combined Pitch-Plunge & Pure Plunge \\
\hline$\alpha_{o}\left({ }^{\circ}\right)$ & 8 & 8 \\
\hline$\theta_{o}\left({ }^{\circ}\right)$ & -8.42 & 0 \\
\hline$h_{o}$ & 0.5 & 0.5 \\
\hline$\lambda$ & 0.6 & 0 \\
\hline$S t$ & 0.08 & 0.08 \\
\hline$k$ & 0.25 & 0.25 \\
\hline$\varphi$ & 0.25 & -- \\
\hline
\end{tabular}

In the Combined Pitch-Plunge case the effective angle of attack oscillates harmonically between $2.53^{\circ}$ to $14.47^{\circ}$, including the static stall angle of attack of $\sim 11$ degrees $^{7}$. Flow visualization and PIV velocity field measurements show that in this case the flow has only small separation region on the suction side of the airfoil during the downstroke portion of the cycle $(\mathrm{t} / \mathrm{T} \sim 0-0.5) .{ }^{5}$ In contrast in the Pure Plunge case the effective angle of attack oscillates harmonically between $-5^{\circ}$ to $23^{\circ}$. In this case the maximum angle of attack is well above steady flow stall conditions. In this case flow visualization and PIV velocity field measurements show formation of a leading edge vortex during the downstroke and full reattachment in the upstroke motion. ${ }^{5}$ Because of the inaccuracy of the axial force measurement, the lift coefficient reported below is derived from the normal force measured by the load cell only without correcting for the incidence angle of the airfoil nor the inertia of the model.

Figure 6 shows the instantaneous normal force during a few oscillation periods. Figure 6(a) is for the combined pitch-plunge motion. In this case the normal force oscillates between 0.5 and $1.5 \mathrm{lbf}$. For pure plunge motion shown in Figure 6(b) the force oscillates between slightly negative and $2.6 \mathrm{lbf}$. Cycle-to-cycle fluctuations are present although the magnitude is relatively small 


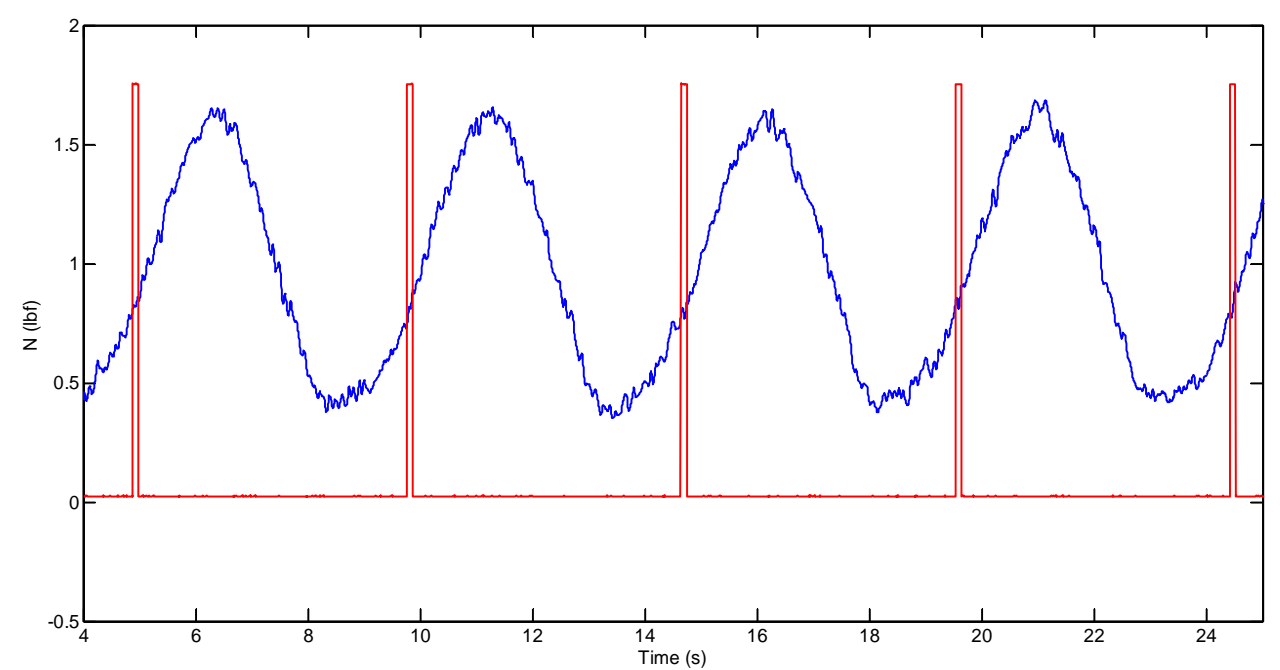

(a)

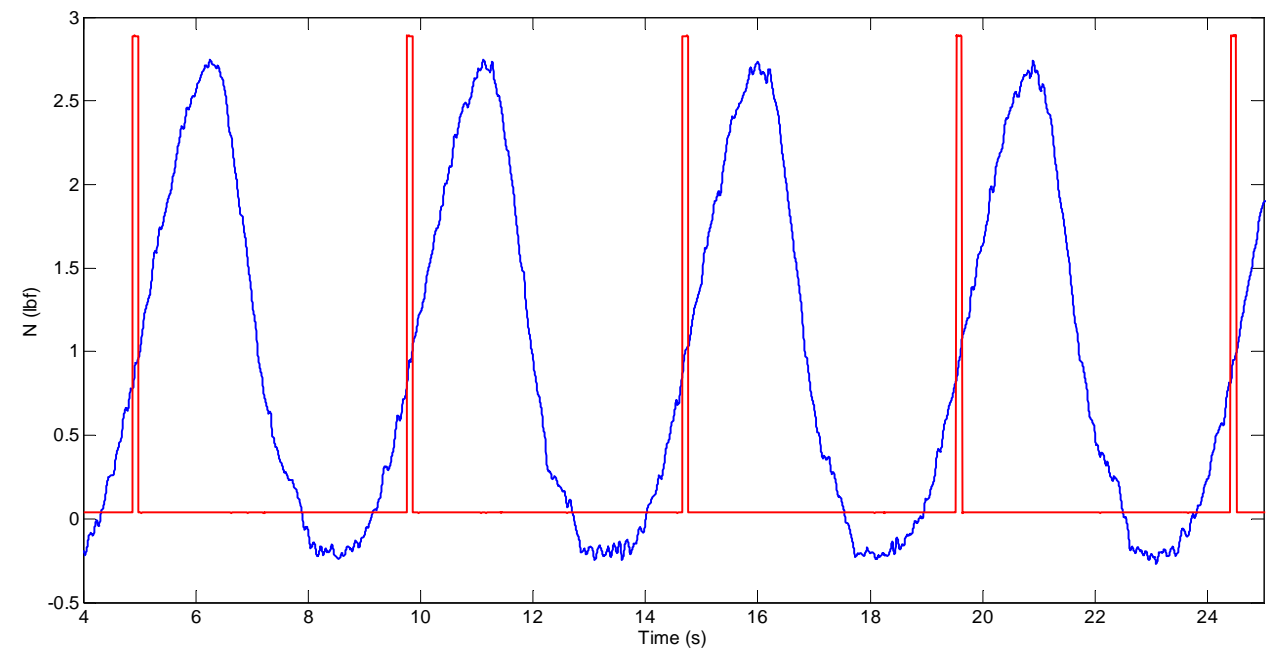

(b)

Figure 6. Instantaneous normal force acting on the SD7003 airfoil at $\mathrm{Re}=\mathbf{6 0 , 0 0 0}$. (a) Combined Pitch-Plunge airfoil motion, (b) Pure plunge.

Figure 7 plots the lift coefficient as a function of phase for the two motions kinematics. These are phaseaveraged results computed for a sample of 198 cycles. Also shown in the figure are the standard deviation of the cycle-to-cycle fluctuations which for these measurement is a good estimate of the $95 \%$ confidence interval. The pure plunge case show significantly larger lift coefficients compared to the combined pitch-plunge case. The maximum lift coefficient is found approximately at phase of 0.25 that corresponds to the maximum effective angle of attack in both cases. The evolution of the lift coefficient during the cycle presents some distinct features in the two cases. For the combined pitch-plunge case the oscillation is approximately sinusoidal, while for the pure plunging case the build-up of lift coefficient from phase 0.75 to 1 , and from 0 to 0.25 is more gradual that the decrease in lift coefficient from phase 0.25 to 0.75 . Also in the pure plunge case the lift coefficient increases more rapidly between phase 0.2 and 0.25 just before reaching the maximum value. This feature can be attributed to the formation of the leading edge vortex. The rapid reduction of lift coefficient that follows is consistent with detachment of the leading edge vortex. Finally at phase 0.55 there is a change in the slope of the lift coefficient that is probably associate with the formation of trailing edge vortex ${ }^{5}$. 


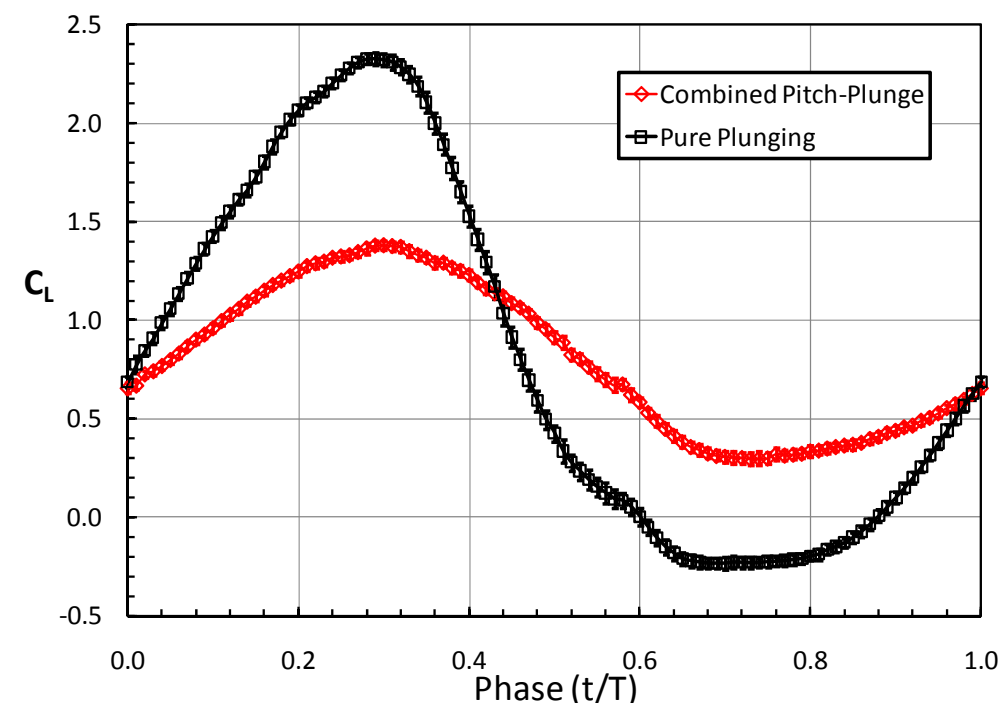

Figure 7. Lift coefficient as a function of phase for SD7003 airfoil at $\mathrm{Re}=\mathbf{6 0 , 0 0 0}$. Error bars, which are approximately the size of the symbols, are estimates of the $95 \%$ confidence interval.

Figure 8 plots the lift coefficient as a function of effective angle of attack for combined pitch-plunge motions. Also shown in the figure are Theodorsen's ${ }^{8}$ unsteady linear theory, steady flow data from Figure 5(a) and XFOIL results for steady flow also. Also shown is a line with slope 0.1 corresponding to the steady linear theory prediction. It is clear that the unsteady airfoil motion results is significantly larger lift coefficient than for steady flow conditions. Comparison with Theodorsen's linear theory and the results of flow visualization and PIV measurement suggest that this increase performance is not associated with the formation of a well-defined dynamics stall vortex, but rather the formation of an enclosed recirculation region.

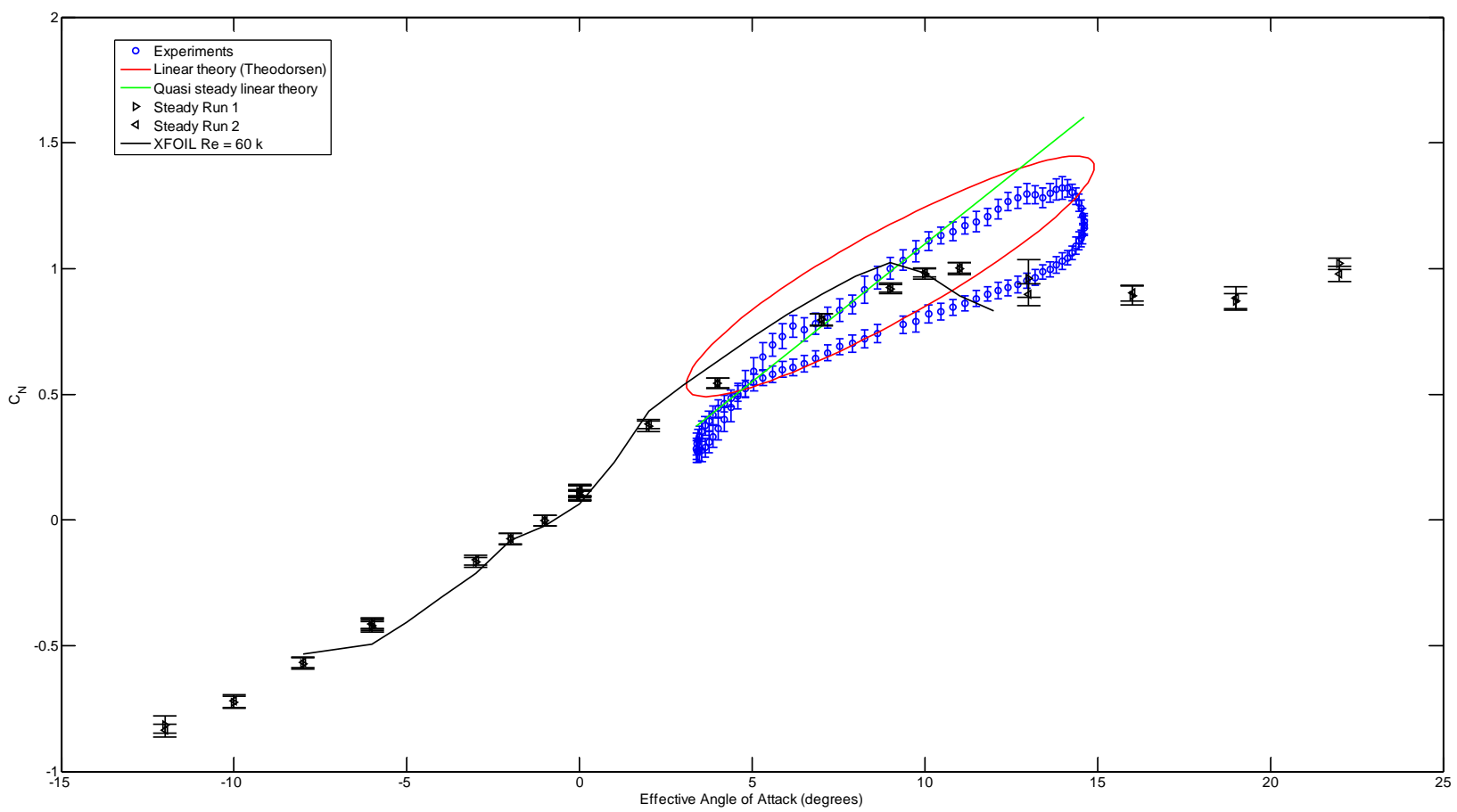

Figure 8. Lift coefficient as a function of angle of attack for the SD7003 airfoil in combined pitch-plunge motion at $R e=60,000$. Error bars give estimates of the $95 \%$ confidence interval.

11

American Institute of Aeronautics and Astronautics 


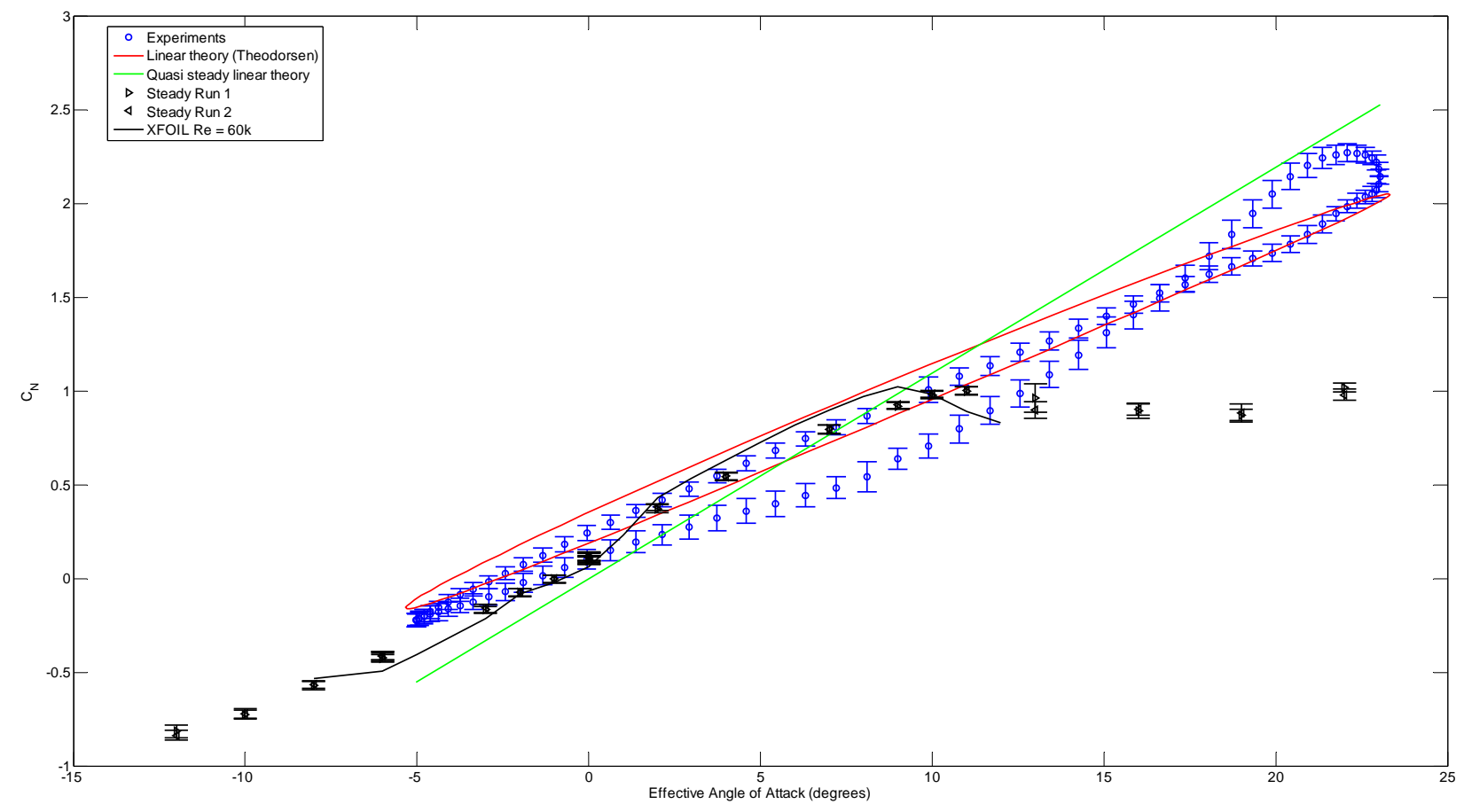

Figure 9. Lift coefficient as a function of angle of attack for the SD7003 airfoil in pure pitch motion at $\operatorname{Re}=$ 60,000 . Error bars give estimates of the $95 \%$ confidence interval.

Figure 9 plots the lift coefficient as a function of effective angle of attack for pure pitch motion. Also shown in the figure are Theodorsen's ${ }^{8}$ unsteady linear theory, steady flow data from Figure 5(a), XFOIL results for steady flow and a straight line with slope 0.1 corresponding to the steady linear theory prediction. As in the previous case the unsteady airfoil motion results in much larger lift coefficient than for steady flow conditions. In contrast with the combined pitch plunge case, the measured lift coefficient at high angle of attack is higher than Theodorsen's linear theory prediction suggesting that the leading edge vortex produces much larger lift force, similar to a dynamic stall vortex. Also during the upstroke phase the lift coefficient is significantly less than Theodorsen's unsteady linear theory prediction. Again the detachment of the leading edge vortex may account for the reduced lift coefficient.

\section{Concluding Remarks}

A new load cell for measuring force at the AFRL Free-surface Horizontal Water Tunnel has been developed. Fiber Bragg Gratings are used to measure strain in the load cell flexures. The technique present significan advantages in terms of noise reduction and simplicity of installation, and challenges in temperature compensation, load cell calibration. New designs and operating procedures are being developed to address the limitations and to improve dynamic load corrections. These new design have significantly improve axial force resolution.

The load cell has been used to measure lift, drag and pitching moment coefficient for a SD 7003 airfoil at Reynolds number 60,000 under steady and unsteady flow condition. The steady flow results show very good agreement with well-established results. For the unsteady flow results the data suggest relatively small departure from unsteady linear theory results at the relatively small effective angle of attack oscillation of the combined pitchplunge case. This is somewhat surprising since the airfoil motions tested are large amplitude, well beyond expected values for application of linear theory. The unsteady flow results for the large effective angle of attack oscillation in the pure plunge case show the increase of lift coefficient due to the leading edge vortex. This increase is well above the unsteady linear theory prediction. Features in the lift coefficient cycle evolution have also been identified associated with the detachment of the leading edge vortex and the formation of the trailing edge vortex. 


\section{Acknowledgments}

The work has been supported in part by the Air Force Office of Scientific Research's Multidisciplinary University Research Initiative (MURI) and by the Michigan/AFRL (Air Force Research Laboratory)/Boeing Collaborative Center in Aeronautical Sciences.

\section{References}

${ }^{1}$ Shyy, W., Lian, Y., Tang, J., Viieru, D., and Liu, H. Aerodynamics of Low Reynolds Number Flyers. Cambridge University Press, 2008.

${ }^{2}$ Ol, M. "Vortical Structures in High Frequency Pitch and Plunge at Low Reynolds Number". AIAA 2007-4233, 2007.

${ }^{3}$ Anderson, J.M., Streitlien, K., Barrett, D.S. and Triantafyllou, M.S., "Oscillating airfoils of high propulsion efficiency" Journal of Fluid Mechanics. Vol. 360, 1998, pp 41-72.

${ }^{4}$ Heathcote, S., Wang, Z., and Gursul, I., "Effect of spanwise flexibility on flapping wing propulsion," Journal of Fluids and Structures, Vol. 24, 2008, pp 183-199.

${ }^{5}$ Ol, M., Bernal, L., Kang, C.-K., and Shyy, W., "Shallow and deep dynamic stall for low Reynolds number airfoils" Experiments in Fluids, Vol. 46, 2009, pp 883-901.

${ }^{6}$ Zhang, L., Zhang, W., and Bennion, I., "In-Fiber Grating Optic Sensors", in Fiber Optic Sensors, Ed. Yu, F.T.S. and Yin, S., Marcel Dekker, Inc., 2002, pp 123-181

${ }^{7}$ Ol M, McAuliffe BR, Hanff ES, Scholz U, and Kaehler Ch. "Comparison of laminar separation bubble measurements on a low Reynolds number airfoil in three facilities" AIAA-2009-5149

${ }^{8}$ Theodorsen, T., "General Theory of Aerodynamic Instability and the Mechanism of Flutter," N.A.C.A. Report 496, 1935 